\title{
ChemComm
}

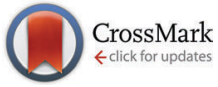

Cite this: Chem. Commun., 2015, 51, 8801

Received 17th February 2015, Accepted 17th April 2015

DOI: $10.1039 / \mathrm{c} 5 \mathrm{cc} 01488 \mathrm{c}$

www.rsc.org/chemcomm

\section{Online monitoring the isomerization of an azobenzene-based dendritic bolaamphiphile using ion mobility-mass spectrometry $\dagger$}

\author{
Leonhard H. Urner, ${ }^{a}$ Bala N. S. Thota, ${ }^{a}$ Olaf Nachtigall, ${ }^{a}$ Stephan Warnke, \\ Gert von Helden, ${ }^{b}$ Rainer Haag*a and Kevin Page ${ }^{\star a b}$
}

Ion mobility-mass spectrometry was used to obtain detailed information about the kinetics of the light-induced cis/trans isomerization process of a new supramolecular azobenzene-based bolaamphiphile. Further experiments revealed that the investigated light-induced structural transition dramatically influences the aggregation behaviour of the molecule.

Self-assembly of amphiphilic molecules is one of the most commonly observed phenomena in nature. Inspired by the high diversity of the underlying driving forces ${ }^{1-3}$ and the complexity of the resulting materials, several synthetic amphiphilic molecules have been introduced over the last few decades. One of these evolving classes are so-called bolaamphiphiles - amphiphiles which are characterized by two polar head groups connected through an alkyl spacer. ${ }^{4}$ Their structure is reminiscent to those of membrane lipids found in archaea bacteria whose high assembly stability is one of the foundations for their colonization of tiny ecological niches, which are hostile to life for all other organisms. ${ }^{5,6}$ As such, purely native systems are of rather limited use when a controlled assembly and disassembly is crucial for the intended application. A common route to circumvent this problem is to introduce moieties which modulate the physicochemical properties of the molecule in response to external stimuli. In this context, especially photo-responsive moieties like azobenzenes, spiropyrans or diarylethenes have recently attracted increased attention. ${ }^{7-9}$ In the case of azobenzene derivatives, the absorption of photons is typically accompanied by a change in geometry and polarity, which in turn can be used to control aggregation processes. ${ }^{10,11}$ Due to the complexity of the formed aggregates, it is often challenging to characterize

\footnotetext{
${ }^{a}$ Freie Universität Berlin, Department of Biology, Chemistry and Pharmacy,

Takustrasse 3, Berlin, Germany.E-mail: haag@chemie.fu-berlin.de,

kevin.pagel@fu-berlin.de

${ }^{b}$ Fritz Haber Institute of the Max Planck Society, Department of Molecular Physics,

Faradayweg 4-6, Berlin, Germany

$\dagger$ Electronic supplementary information (ESI) available: Experimental and synthetic procedures and characterization data employed in this work are given. See DOI: $10.1039 / \mathrm{c} 5 \mathrm{cc} 01488 \mathrm{c}$
}

the assembly kinetics of switchable azobenzene-based materials in full detail. A promising method to provide an additional dimension of molecular level information is ion mobility-mass spectrometry (IM-MS). In IM-MS ions are separated according to the time that is required to traverse a cell filled with inert neutral gas when a weak electric field is applied. This drift time not only depends on the mass-to-charge $(\mathrm{m} / \mathrm{z})$ ratio of the ions, but also on their size and shape, which effectively allows the separation of isomers. ${ }^{12-14}$

Here we present for the first time that IM-MS can be used for the direct online-monitoring of the light induced molecular shape transition of a new azobenzene-based dendritic bolaamphiphile. To do so, the molecule was irradiated with light directly at the nanoelectrospray ionization (nESI) interface followed by a timeresolved analysis of resulting cis/trans ratio in the gas phase using IM-MS. Further TEM measurements revealed that the lightinduced structural transition dramatically influences the aggregation behaviour.

The structure of the symmetric bolaamphiphile G1azoG1 6 focused here is shown in Scheme 1. The synthesis was performed in five steps (for details see ESI $\dagger$ ). First, compound 1 was treated with propargyl bromide under basic conditions to generate the symmetric $O$-propargylated product 2. Coupling of the azido ester with compound 2 via "click chemistry" directly lead to the diester 3. Subsequently, the diester was hydrolyzed in ethanol under basic conditions and the resulting acid $\mathbf{4}$ was converted into the symmetric acid chloride and coupled with $\operatorname{protG}_{1}-\mathrm{NH}_{2}$ under basic conditions to give compound 5. Finally, the acetal protecting groups of compound $\mathbf{5}$ were removed with an excess of trifluoroacetic acid to quantitatively yield compound 6 .

Due to their different absorption properties, cis/trans isomers of G1azoG1 6 can be distinguished by UV/VIS spectroscopy. The absorption spectrum of trans G1azoG1 6 exhibits a strong $\pi \rightarrow \pi^{*}$ absorption band (356 $\mathrm{nm}$ ) and a weaker $\mathrm{n} \rightarrow \pi^{*}$ absorption band $(410 \mathrm{~nm})$ at longer wavelengths. After conversion into the cis isomer via irradiation with UV light at $366 \mathrm{~nm}$, on the other hand, the $\pi \rightarrow \pi^{*}$ transition is perceptibly blue shifted to $318 \mathrm{~nm}$, while a red shift is observed for the $\mathrm{n} \rightarrow \pi^{*}$ transition at $431 \mathrm{~nm}$. 


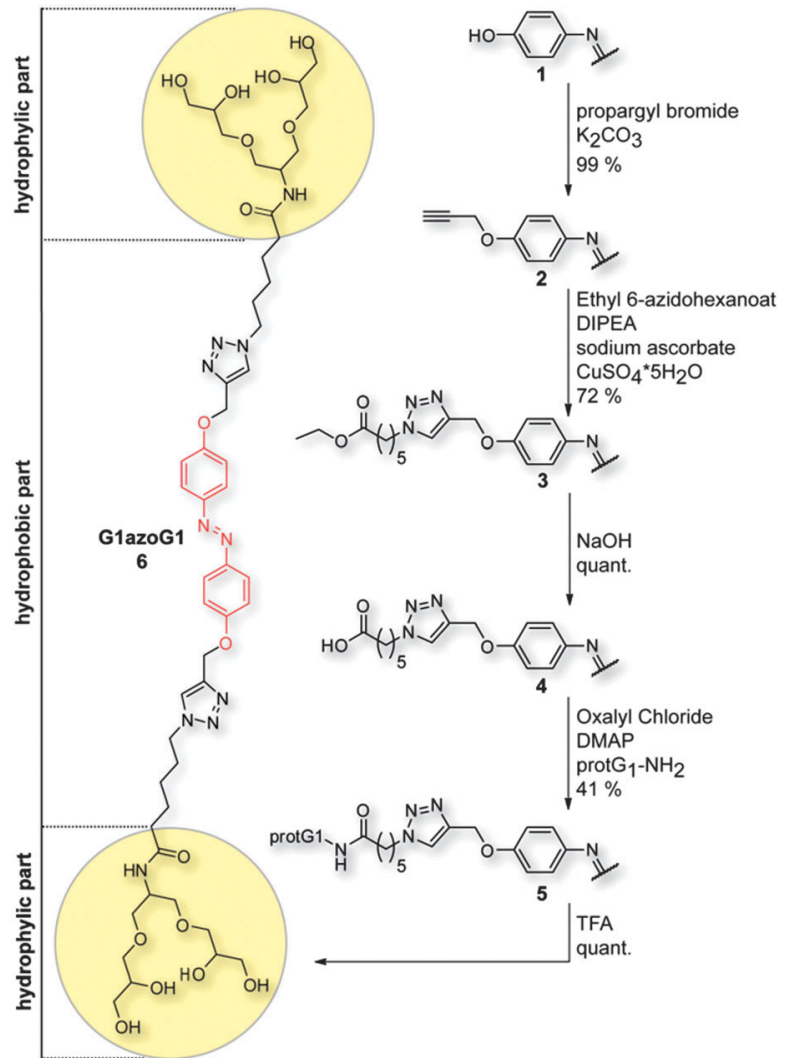

Scheme 1 Synthetic paths to G1azoG1 $\mathbf{6}$. The half drawings of 1-5 are symmetric to the rest of the molecule.

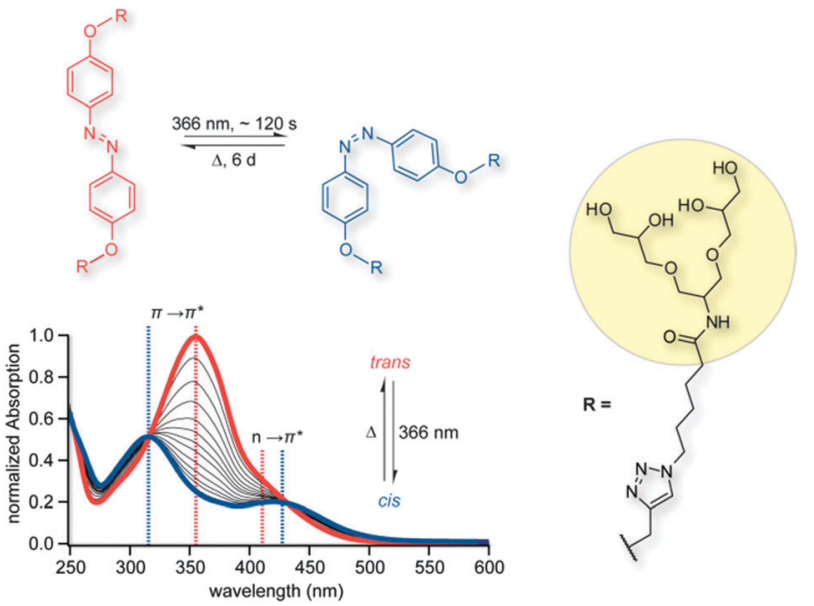

Fig. 1 UV/VIS absorption spectra showing the trans to cis conversion of G1azoG1 6. The photoinduced isomerization is completed after $120 \mathrm{~s}$, whereas the thermal cis to trans conversion takes $6 \mathrm{~d}$ when stored in the dark. The side chains of GlazoG1 6 are abbreviated by an " $\mathrm{R}$ ".

In order to determine the time that is required to reach the maximum saturation of the cis form, UV/VIS spectra were recorded after stepwise irradiation at $366 \mathrm{~nm}$. To do so, the sample was exposed to light for $5 \mathrm{~s}$ and a spectrum was measured. Subsequently, the procedure was repeated until the conversion was complete; a full saturation regarding the cis state was observed after approximately $120 \mathrm{~s}$ (blue line, Fig. 1) under certain experimental conditions (see Table ES1, ESI $\dagger$ ). Moreover, a fully converted sample thermally reconverts from the cis to the trans form within approximately six days when stored in the dark (Fig. ES1, ESI $\dagger$ ). Further attempts to differentiate between both structural isomers in solution using DOSY experiments failed, because here both isoforms exhibit similar diffusion coefficients (Fig. ES2, ESI $\dagger$ ).

For online-monitoring of the cis/trans isomerization of G1azoG1 6 via IM-MS an in-house-built drift tube (DT) IM-MS instrument was used (for details see ESI $\dagger$ ). ${ }^{15,16}$ The source region of this instrument was equipped with two light sources to enable irradiation of the sample prior to ionization (Fig. 2a). Ions are generated via nESI using borosilicate capillaries prepared in-house using a previously described procedure. ${ }^{17}$ The Pd/Pt coated, but still partially transparent capillary was irradiated with: (a) a mercury vapour UV lamp $(\lambda=366 \mathrm{~nm})$ to induce trans to cis isomerization or (b) a tungsten filament lamp $\left(T=3300 \mathrm{~K}, \lambda_{\max } \approx 880 \mathrm{~nm}\right)$ to induce cis to trans isomerization (Fig. 2a). As a result, the cis/trans equilibrium within the capillary can be monitored directly in a time-resolved manner via IM-MS analysis.

Regardless of the configuration, mixtures of singly and doubly charged sodiated and/or protonated ions are observed after nESI of G1azoG1 6 (Fig. ES3, ESI $\dagger$ ). For singly charged ions only one peak is observed in the IM-MS arrival time distribution (ATD). This indicates, that cis and trans isoforms of singly charged G1azoG1 6 ions adopt very similar structures in the gas phase. Due to the structural flexibility of the molecule, this observation is not surprising since the dendric side chains are likely to collapse in the gas phase to solvate the charge. Coulomb repulsion in doubly charged ions, on the other hand, leads to two distinct conformations, which can be clearly separated by IM-MS as shown exemplarily for doubly sodiated ions in Fig. 2b. After irradiation of the sample inside the nESI capillary at $366 \mathrm{~nm}$, the species at shorter drift time is dominant with only a minor content of the isomer with longer drift time. Subsequent irradiation of the sample with visible light, on the other hand, leads to a complete inversion of the intensities, with the species at longer drift time now being the major component. This leads to the conclusion that the peak at shorter drift time can be assigned to the cis isomer and the peak at larger drift time to the trans isomer. Multiple repetition of the switching process revealed that the isomerization process is completely reversible. Generally, a good separation between the two isoforms was observed for all doubly charged ions, i.e. $[\mathrm{M}+2 \mathrm{H}]^{2+},[\mathrm{M}+2 \mathrm{Na}]^{2+}$ and $[\mathrm{M}+\mathrm{NaH}]^{2+}$. The relative abundance of the individual doubly charged species, however, was shifted considerably. This is very likely a result of a different ESI ionization efficiency, which is known to strongly depend on the size and accessibility of the molecule. In order to take that into account in our analysis, each observed adduct was considered as a part of an ensemble of cis or trans molecules in solution. The absolute number of all observed cis or trans species was summed up and divided by the total intensity of all doubly charged ions. Subsequently, the relative populations of the cis and trans isomers were normalized and plotted against the 
a)

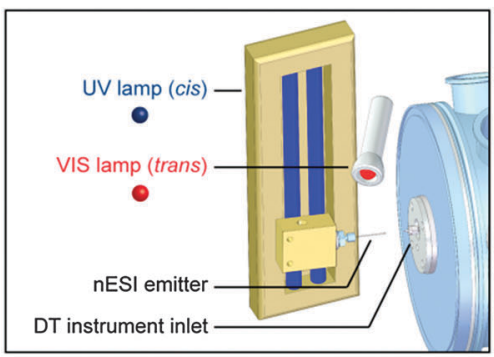

c)

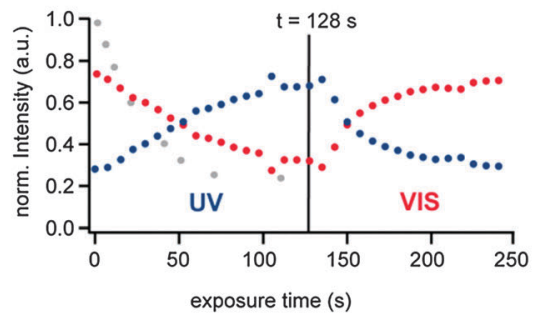

b)

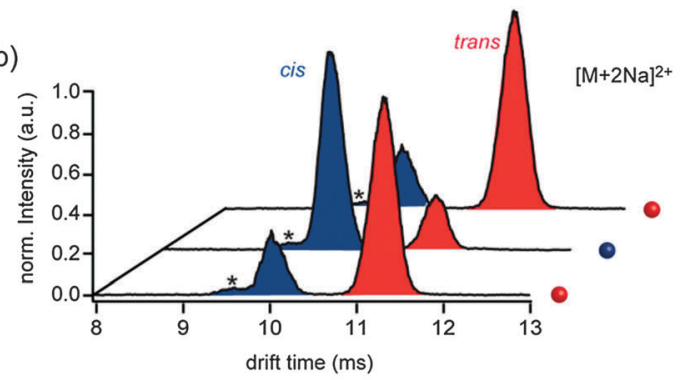

d)

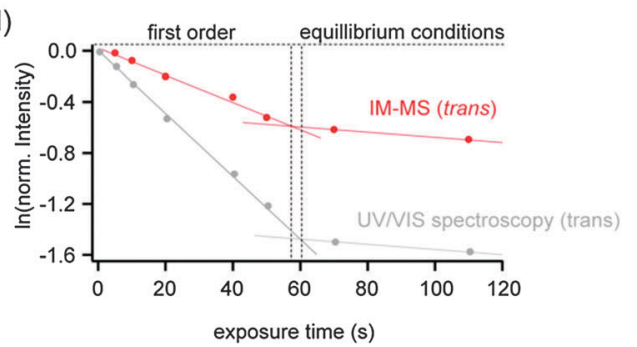

Fig. 2 Ion mobility-mass spectrometry (IM-MS) of G1azoG1 6. (a) Modified source region of the utilized DT IM-MS instrument. (b) Arrival time distributions (ATDs) of $[M+2 \mathrm{Na}]^{2+}$ ions after irradiation at $\lambda=366 \mathrm{~nm}$ (blue dot) or with visible light (red dot), respectively. Peaks resulting from neighbouring masses are labelled with an asterisk. (c) Relative intensities of the cis- and trans populations plotted against exposure time. The normalized intensity of trans G1azoG1 6 observed via UV/VIS spectroscopy is shown as grey circles for comparison. (d) Logarithmic plot of the normalized intensity of trans G1azoG1 6 at $366 \mathrm{~nm}$ as a function of exposure time determined by IM-MS (red circles) and UV/VIS spectroscopy (grey circles).

exposure time at $366 \mathrm{~nm}$. As shown in Fig. 2c the relative content of cis isomer (blue dots) steadily increases upon irradiation at $366 \mathrm{~nm}$ until saturation is reached after approx. $130 \mathrm{~s}$. Subsequent irradiation with visible light lead to a rapid depletion of the cis form. For comparison, also the intensities observed from the trans absorption band via UV/VIS spectroscopy are shown (Fig. 2c, grey dots). The general shape of the curves is surprisingly similar.

In order to also obtain information on the underlying kinetics, the intensities of trans G1azoG1 6 observed by IM-MS and UV/VIS spectroscopy were fitted to a logarithmic first order exponential equation (Fig. 2d). The linear sections observed for the first $60 \mathrm{~s}$ clearly indicate a monomolecular reaction with the reaction rate of the trans to cis isomerization exclusively depending on the concentration of molecules in the trans state. The increasing concentration of molecules in the cis state is affecting the cis to trans reconversion. This is reflected in a clear deviation from a monomolecular reaction after $60 \mathrm{~s}$. Surprisingly, both techniques IM-MS as well as UV/VIS analysis perfectly agree with the observable mechanistic change from first order to equilibrium conditions. However, the fact that the observed transition between first order kinetics and equilibrium conditions occurs almost simultaneously in both methods is purely coincidental (for details see Table ES1, ESI $\dagger$ ).

In addition to the analysis of the isolated monomers, the influence of the light induced structural changes on the aggregation behaviour was studied. First, the critical aggregation concentration (cac) was determined before and after irradiation at $366 \mathrm{~nm}$ using the pendant drop method (for details see ESI $\dagger) .{ }^{18,19}$ The cac of the non-irradiated trans form $\left(\right.$ cac $_{\text {trans }}=$ $6.3 \times 10^{-5} \mathrm{M}$ ) was found to be at least 10 times lower than the cac of the of the irradiated cis form $\left(\mathrm{cac}_{c i s}=5.7 \times 10^{-4} \mathrm{M}\right)$. This trend is expected, since the dipole moment of the azobenzene is known to increase considerably during the trans to cis isomerization. ${ }^{10,20}$ Furthermore, the solubility increases and the amphiphilic nature of G1azoG1 6 is reduced. Secondly, the morphology of the aggregates was studied using TEM. To do so, aliquots of one and the same aqueous solution of G1azoG1 6 with a concentration of $10^{-4} \mathrm{M}$ (i.e. above cac trans $_{\text {, but below }}$ $\mathrm{cac}_{\text {cis }}$ ) were studied before and after irradiation at $366 \mathrm{~nm}$. In the non-irradiated trans form, G1azoG1 6 forms regular twisted tapes with lengths up to several micrometers and widths of approximately $20 \mathrm{~nm}$ (Fig. 3a and c). Interestingly, the observed aggregates exhibit a constant width of around $5 \mathrm{~nm}$, which agrees well with the length of the monomer in a stretched arrangement. As a result, the overall structure of the twisted tape along the longitudinal axis can be explained using a simple model structure (Fig. 3d). TEM after irradiation at $366 \mathrm{~nm}$ for $30 \mathrm{~min}$, on the other hand, showed the complete breakup of the twisted tapes (Fig. 3b). Therefore, it can be concluded that the trans form of G1azoG1 6 forms well-ordered, tape-like structures at $10^{-4} \mathrm{M}$ while the cis form shows no aggregation at these conditions. As a result, the tendency to form higher order aggregates at $10^{-4} \mathrm{M}$ can be switched on and off simply by irradiation with light at $366 \mathrm{~nm}$.

In summary, our data show that the photoinduced trans to cis isomerization of a new azobenzene-based dendritic bolaamphiphile can be followed online via direct irradiation of the sample at the nESI interface. The comparison between IM-MS and UV/VIS analysis showed that both methods perfectly agree with the observed mechanistic change from first order to equilibrium conditions. Moreover, TEM measurements revealed that the light-induced structural transition dramatically influences the aggregation behaviour of the investigated bolaamphiphile. Our experiments show that the breakup of the observed 

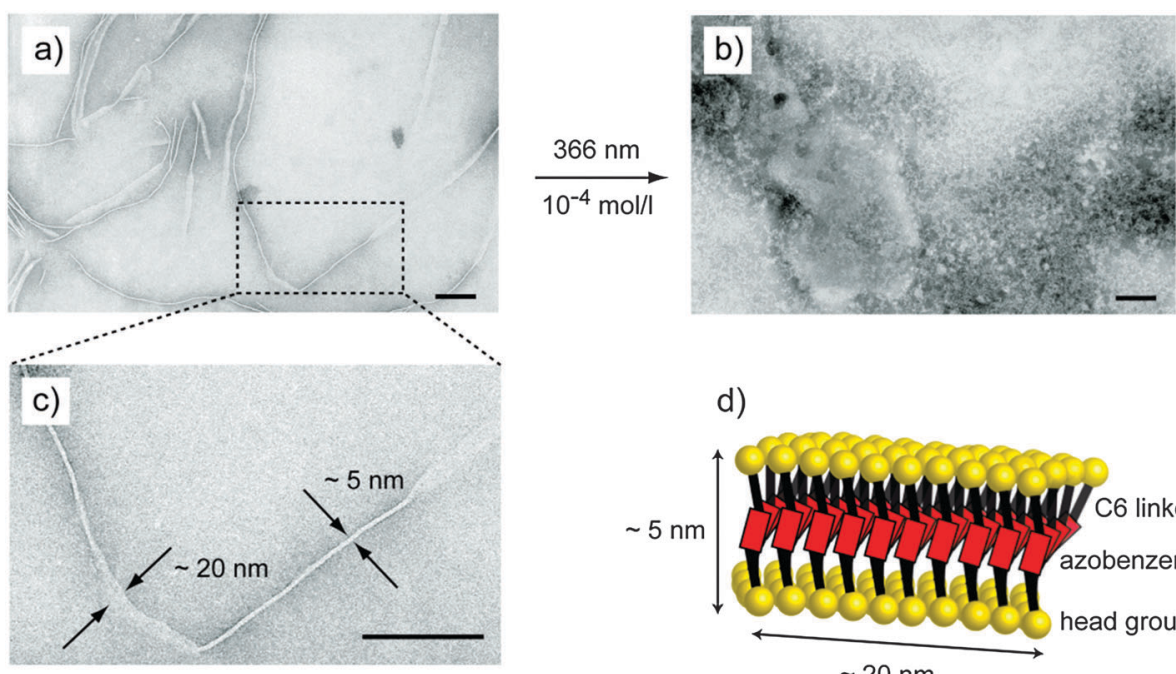

.

tape-like structures can be induced simply by irradiation at $366 \mathrm{~nm}$.

This project was supported by the DFG (SFB 658). Andrea Schulz is gratefully acknowledged for performing the TEM measurements. The authors thank Michael T. Bowers for helpful discussions and continuous support and also Andreas Schäfer is gratefully acknowledged for performing the NMR measurements.

\section{Notes and references}

1 T. Rehm and C. Schmuck, Chem. Commun., 2008, 801-813.

2 F. Huang, R. O'Reilly and S. C. Zimmerman, Chem. Commun., 2014, 50, 13415-13416.

3 A. Lützen, CHEMKON, 2007, 14, 123-130.

4 J. H. Fuhrhop and T. Wang, Chem. Rev., 2004, 104, 2901-2937.

5 G. D. Sprott, J. Bioenerg. Biomembr., 1992, 24, 555-566.

6 A. Gambacorta, A. Gliozzi and M. De Rosa, World J. Microbiol. Biotechnol., 1995, 11, 115-131.

7 M. M. Russew and S. Hecht, Adv. Mater., 2010, 22, 3348-3360.

8 S. Peng, Q. Guo, P. G. Hartley and T. C. Hughes, J. Mater. Chem., 2014, 2, 8303-8312.
9 T. Sendai, S. Biswas and T. Aida, J. Am. Chem. Soc., 2013, 135, 11509-11512.

10 S. Hecht, Small, 2005, 1, 26-29.

11 C. Stoffelen, J. Voskuhl, P. Jonkheijm and J. Huskens, Angew. Chem., 2014, 126, 3468-3472.

12 J. J. Santos, S. H. Toma, P. M. Lalli, M. F. Riccio, M. N. Eberlin, H. E. Toma and K. Araki, Analyst, 2012, 137, 4045-4051.

13 J. Thiel, D. Yang, M. H. Rosnes, X. Liu, C. Yvon, S. E. Kelly, Y. F. Song, D. L. Long and L. Cronin, Angew. Chem., Int. Ed. Engl,, 2011, 50, 8871-8875.

14 Á. Révész, D. Schröder, T. A. Rokob, M. Havlík and B. Dolenský, Angew. Chem., Int. Ed., 2011, 50, 2401-2404.

15 P. R. Kemper, N. F. Dupuis and M. T. Bowers, Int. J. Mass Spectrom., 2009, 287, 46-57.

16 S. Warnke, C. Baldauf, M. T. Bowers, K. Pagel and G. von Helden, J. Am. Chem. Soc., 2014, 136, 10308-10314.

17 H. Hernandez and C. V. Robinson, Nat. Protoc., 2007, 2, 715-726.

18 C. Kordel, C. S. Popeney and R. Haag, Chem. Commun., 2011, 47, 6584-6586.

19 F. M. Menger, A. L. Galloway and M. E. Chlebowski, Langmuir, 2005, 21, 9010-9012.

20 H. Yan, Y. Long, K. Song, C.-H. Tung and L. Zheng, Soft Matter, 2014, 10, 115-121. 